\title{
Tigecycline Therapy for Nosocomial Pneumonia due to Carbapenem-Resistant Gram-Negative Bacteria in Critically Ill Patients Who Received Inappropriate Initial Antibiotic Treatment: A Retrospective Case Study
}

\author{
Xiaomai Wu, ${ }^{1}$ Yefei Zhu, ${ }^{1}$ Qiuying Chen, ${ }^{2}$ Liuyang Gong, ${ }^{1}$ Jian Lin, ${ }^{1}$ \\ Dongqing Lv, ${ }^{1}$ and Jiaxi Feng ${ }^{1}$ \\ ${ }^{1}$ Department of Respiratory Medicine, Taizhou Hospital of Zhejiang Province, Linhai 317000, China \\ ${ }^{2}$ Operation Department, Taizhou Hospital of Zhejiang Province, Linhai 317000, China
}

Correspondence should be addressed to Xiaomai Wu; wu.xiaomy@aliyun.com

Received 8 August 2016; Revised 9 November 2016; Accepted 14 November 2016

Academic Editor: Paola Di Carlo

Copyright (C) 2016 Xiaomai Wu et al. This is an open access article distributed under the Creative Commons Attribution License, which permits unrestricted use, distribution, and reproduction in any medium, provided the original work is properly cited.

\begin{abstract}
Background. Nosocomial pneumonia due to carbapenem-resistant Gram-negative bacteria (CRGNB) is a growing concern because treatment options are limited and the mortality rate is high. The effect of tigecycline (TGC) on nosocomial pneumonia due to CRGNB in patients who have received inappropriate initial empiric antibiotic treatment (IIAT) is unclear. Therefore, this study aimed to examine the effect of TGC on nosocomial pneumonia due to CRGNB in critically ill patients who had received IIAT. Methods. A retrospective study was conducted in an adult respiratory intensive care unit. Data were obtained and analyzed for all patients who were treated with TGC $\geq 3$ days for microbiologically confirmed nosocomial pneumonia due to CRGNB and had experienced initial antibiotic failure. Clinical and microbiological outcomes were investigated. Results. Thirty-one patients with hospital-acquired pneumonia or ventilator-associated pneumonia were included in the study. The majority of the responsible organisms were carbapenem-resistant Acinetobacter baumannii (67.7\%), followed by Klebsiella pneumoniae (16.1\%) and Escherichia coli (9.7\%). Twenty patients were treated with high-dose TGC therapy (100 mg every $12 \mathrm{~h}$ after a $200 \mathrm{mg}$ loading dose), and the others received a standard-dose therapy ( $50 \mathrm{mg}$ every $12 \mathrm{~h}$ after a $100 \mathrm{mg}$ loading dose). The duration of TGC therapy was $14.3 \pm 2.8$ days. The global clinical cure rate and the microbiological eradication rate were $48.4 \%$ and $61.3 \%$, respectively. The overall ICU mortality rate was $45.2 \%$. A higher score on the Acute Physiology and Chronic Health Evaluation II and a longer duration of IIAT were associated with clinical failure. High-dose TGC therapy had a higher clinical success rate $[65.0 \%(13 / 20)$ versus $18.2 \%$ (2/11), $P=0.023]$ and a lower ICU mortality rate $[30.0 \%(6 / 20)$ versus $72.7 \%(8 / 11), P=0.031]$ than the standard-dose therapy. Conclusions. TGC, especially a high-dose regimen, might be a justifiable option for critically ill patients with nosocomial pneumonia due to CRGNB who have received IIAT when the options for these patients are limited.
\end{abstract}

\section{Introduction}

Tigecycline (TGC) is a derivative of tetracycline that is also known as glycycline and that has a broad spectrum of activity [1]. In vitro, TGC exhibits excellent antibacterial activity against most aerobic and anaerobic bacteria, including multidrug-resistant (MDR) and extensively drug-resistant organisms [2-5]. However, a recent systematic review and metaanalysis suggested that TGC is also associated with a higher risk of death than other antibiotics $[6,7]$. The Food and
Drug Administration has therefore warned clinicians about the risks of TGC

Despite these limitations, because of the scarcity of other effective antimicrobials, TGC has been a useful alternative in patients with serious infections caused by MDR organisms, such as carbapenem-resistant Gram-negative bacteria (CRGNB) [8-12].

Nosocomial pneumonia caused by CRGNB continues to be a growing concern, particularly in the intensive care unit (ICU), because it has limited treatment options and is 
associated with a high mortality rate [13-15]. There is a high risk of failure when using initial empiric antibiotic treatments for infections with CRGNB because the pathogens are usually not covered $[14,16,17]$. Although TGC exhibits considerable in vitro efficacy against CRGNB, the clinical effects of TGC in severe infections caused by CRGNB are controversial [2-7].

The effect of TGC on nosocomial pneumonia due to CRGNB in critically ill patients who have received inappropriate initial empiric antibiotic treatment (IIAT) has not been previously reported. Therefore, this retrospective clinical case study was aimed at assessing the effect of TGC on these patients.

\section{Materials and Methods}

This study was conducted in an eight-bed adult respiratory ICU in a tertiary care hospital and was approved by the Institutional Medical Ethics Review Board of Taizhou Hospital of Zhejiang Province. The need for informed consent was waived because of its retrospective design.

All of the patients included in this study were admitted to the respiratory ICU between 1 January 2013 and 31 December 2015. The inclusion criterion was the administration of TGC for microbiologically documented nosocomial pneumonia caused by CRGNB after any failed initial empiric antibiotic treatment. TGC treatment needed to be provided for at least 3 days.

The data were collected from patient medical records and hospital databases. Clinical data included demographics, underlying diseases, clinical and laboratory findings, microbiological results, Acute Physiology and Chronic Health Evaluation II (APACHE II) scores, and Sequential Organ Failure Assessment (SOFA) scores at admission to the ICU. Data related to information regarding the type and duration of antibiotics used before TGC treatment, the dose and duration of TGC therapy, concomitant antibiotics, clinical and microbiological responses, and ICU mortality were also collected.

2.1. Definitions. In this study, nosocomial pneumonia included hospital-acquired pneumonia (HAP), healthcare-associated pneumonia, and ventilator-associated pneumonia (VAP), which were defined according to the guidelines of the American Thoracic Society and Infectious Diseases Society of America [18]. A diagnosis of pneumonia was made on the basis of abnormal radiographic results (including new or persistent focal infiltrates or a diffuse lung injury pattern) along with one or more of the following criteria: (1) fever or leukocytosis/leukopenia; (2) respiratory failure requiring mechanical ventilation; or (3) at least two of the following: cough, dyspnea, tachypnea, pleuritic chest pain, auscultatory findings of rales or evidence of consolidation, hypoxemia, and purulent sputum or a change in sputum characteristics.

IIAT was defined as antibiotic therapy administered prior to a determination of the infectious pathogens and a later determination that the pathogens were uncovered by the antibiotics. Failed initial empiric antibiotic treatment was defined as meeting one or more of the following criteria after $\geq 48$ h of treatment: (1) the clinical manifestations of pneumonia were not improved; (2) respiratory failure or other organ dysfunction was present or worsened due to pneumonia, (3) laboratory tests indicated deterioration of leukocyte, neutrophil, C-reactive protein, and procalcitonin levels; or (4) a chest radiograph showed progression. Microbiological eradication was defined as negative culture results for the original pathogens during or after the course of TGC therapy.

Responses to treatment were defined as either clinically successful or unsuccessful. Successful treatment was defined as the complete resolution of the infection-related abnormalities by the end of TGC therapy. Improvement in signs on chest radiography was also required for VAP. The criteria for unsuccessful treatment included the persistence of the initial signs of infection that required a change in antibiotic therapy, the reappearance of the initial signs of infection, or infectionrelated death.

2.2. Microbiology Analysis. Quantitative or semiquantitative bacterial cultures were obtained from the bronchoalveolar lavage (BAL), bronchoscopic aspirate, endotracheal aspirate, or qualified sputum specimens. Strains and antimicrobial susceptibilities were identified using the VITEK ${ }^{\circledR} 2$ system (bioMérieux, Marcy-l'Etoile, France). The Clinical and Laboratory Standards Institute criteria published in 2012 were used to interpret the results. CRGNB was defined as carbapenemnonsusceptible ( $\mathrm{MIC} \geq 2 \mathrm{mg} / \mathrm{L}$ ) and extended-spectrum cephalosporin-resistant Gram-negative bacteria. With regard to tigecycline, susceptibility was interpreted according to the breakpoints approved by the US Food and Drug Administration. The isolates were considered susceptible to TGC if the MIC was $\leq 2 \mathrm{mg} / \mathrm{L}$, intermediate if the $\mathrm{MIC}=4 \mathrm{mg} / \mathrm{L}$, and resistant if the MIC $\geq 8 \mathrm{mg} / \mathrm{L}$.

2.3. Statistical Analysis. The Shapiro-Wilk test was used to evaluate the distribution of variables. Continuous variables were assessed using Student's $t$-test or the Mann-Whitney $U$ test, as appropriate, and expressed as the mean \pm standard deviation (SD) or median and interquartile range (IQR). Categorical variables were analyzed using Fisher's exact test and are presented as proportions. A $P$ value $<0.05$ was considered statistically significant. The SPSS v16.0 package (SPSS Inc., Chicago, IL, USA) was used for all statistical analyses.

\section{Results}

3.1. Demographic Characteristics of the Patients. During the study period, 1085 patients were admitted to the respiratory ICU. Two hundred and ninety-eight patients were diagnosed with HAP, including 95 patients with VAP. The bacterial pathogens were confirmed in 65.8\% (196/298) of these cases, and the initial empirical antibiotic treatment was found to be inappropriate in $37.8 \%(74 / 196)$ of the patients. Patients infected with CRGNB accounted for $71.6 \%$ (53/74) of the cases with IIAT. Among these, 31 cases that were treated with TGC as a salvage therapy were included in the study (Figure 1). The demographics and clinical characteristics of the study patients are shown in Table 1.

3.2. Microbiological Characteristics and Antibiotic Regimens. Among the 31 included patients, the most frequent causative 
TABLE 1: Demographic and clinical data for the included patients.

\begin{tabular}{|c|c|c|c|c|}
\hline Variable $^{*}$ & $\begin{array}{c}\text { Total } \\
(n=31)\end{array}$ & $\begin{array}{l}\text { Successful cases } \\
\quad(n=15)\end{array}$ & $\begin{array}{l}\text { Unsuccessful cases } \\
(n=16)\end{array}$ & $P$ value ${ }^{\#}$ \\
\hline Age, years, mean (SD) & $74.6(9.4)$ & $74.8(9.6)$ & $74.4(9.8)$ & 0.93 \\
\hline Male, $n(\%)$ & $22(71.0)$ & $13(86.7)$ & $9(56.3)$ & 0.113 \\
\hline APACHE II score, median (IQR) & $19.0(15.0-19.0)$ & $15.0(13.5-19.0)$ & $19.0(18.0-20.0)$ & 0.04 \\
\hline SOFA score, mean (SD) & $3.5(1.1)$ & $3.1(0.8)$ & $3.8(1.2)$ & 0.16 \\
\hline Severe sepsis/septic shock, $n(\%)$ & $11(35.5)$ & $5(33.3)$ & $6(37.5)$ & 1.0 \\
\hline VAP, $n(\%)$ & $10(32.3)$ & $4(26.7)$ & $6(37.5)$ & 0.704 \\
\hline ICU LOS before TGC, day, median (IQR) & $8(1-13)$ & $7(0.5-10.0)$ & $9(6.3-14.5)$ & 0.16 \\
\hline \multicolumn{5}{|l|}{ Comorbidities, $n(\%)$} \\
\hline COPD & $16(51.6)$ & $7(46.7)$ & $9(56.3)$ & 0.724 \\
\hline Bronchiectasis & $3(9.8)$ & $1(6.7)$ & $2(12.5)$ & 1.0 \\
\hline $\mathrm{CHF}$ & $8(25.8)$ & $5(33.3)$ & $3(18.8)$ & 0.433 \\
\hline Diabetes & $5(16.1)$ & $1(6.7)$ & $4(25.0)$ & 0.333 \\
\hline Cerebral apoplexy & $3(9.8)$ & $2(13.3)$ & $1(6.25)$ & 0.60 \\
\hline Immunosuppressive status & $5(16.1)$ & $3(20.0)$ & $2(12.5)$ & 0.654 \\
\hline Comorbidities $>1$ & $17(54.8)$ & $10(66.7)$ & $7(43.8)$ & 0.285 \\
\hline \multicolumn{5}{|l|}{ Responsible pathogens, $n(\%)$} \\
\hline Acinetobacter baumannii & $21(67.7)$ & $9(60.0)$ & $12(75.0)$ & 0.458 \\
\hline Klebsiella pneumoniae & $5(16.1)$ & $3(20.0)$ & $2(12.5)$ & 0.654 \\
\hline Escherichia coli & $3(9.7)$ & $1(6.7)$ & $2(12.5)$ & 1.0 \\
\hline Stenotrophomonas maltophilia & $2(6.5)$ & 0 & $2(12.5)$ & 0.484 \\
\hline \multicolumn{5}{|l|}{ Type of initial empiric antibiotics, $n(\%)$} \\
\hline Cephalosporin & $4(12.9)$ & $1(6.7)$ & $3(18.8)$ & 0.60 \\
\hline $\begin{array}{l}\text { Combination } \beta \text {-lactam } / \beta \text {-lactamase } \\
\text { inhibitor }\end{array}$ & $12(38.7)$ & $6(40.0)$ & $6(37.5)$ & 1.0 \\
\hline Carbapenem & $15(48.4)$ & $10(66.7)$ & $5(31.3)$ & 0.076 \\
\hline Fluoroquinolone & $9(29.0)$ & $4(26.7)$ & $5(31.3)$ & 1.0 \\
\hline Aminoglycoside & $8(25.8)$ & $6(40.0)$ & $2(12.5)$ & 0.113 \\
\hline Glycopeptide & $5(16.1)$ & $1(6.7)$ & $4(25.0)$ & 0.333 \\
\hline $\begin{array}{l}\text { Duration of initial treatment with } \\
\text { antibiotics, day, median (IQR) }\end{array}$ & $13(7-20)$ & $7(7-14)$ & $16(7-20)$ & 0.02 \\
\hline $\begin{array}{l}\text { Duration of treatment with TGC, day, } \\
\text { mean (SD) }\end{array}$ & $14.3(2.8)$ & $12.7(5.9)$ & $15.5(4.2)$ & 0.51 \\
\hline Microbiological eradication, $n(\%)$ & $19(61.3)$ & $13(86.7)$ & $6(37.5)$ & 0.009 \\
\hline ICU mortality, $n(\%)$ & $14(45.2)$ & $1(6.7)$ & $13(81.3)$ & 0 \\
\hline
\end{tabular}

* APACHE II: Acute Physiology and Chronic Health Evaluation II, CHF: chronic heart failure, COPD: chronic obstructive pulmonary disease, ICU: intensive care unit, IMV: invasive mechanical ventilation, IQR: interquartile range, TGC: tigecycline, LOS: length of stay, SD: standard deviation, SOFA: Sequential Organ Failure Assessment, and VAP: ventilator-associated pneumonia.

\#Successful cases versus unsuccessful cases.

organisms were Acinetobacter baumannii $(n=21,67.7 \%)$ and Klebsiella pneumoniae $(n=5,16.1 \%)$, followed by Escherichia coli $(n=3,9.7 \%)$ and Stenotrophomonas maltophilia $(n=2$, $6.5 \%)$. The antimicrobial resistances of the isolates are shown in Table 2.

Carbapenem $(n=15,48.4 \%)$ was the most frequently used initial empiric antibiotic treatment in the cohort, followed by a $\beta$-lactam $/ \beta$-lactamase inhibitor combination and fluoroquinolone (Table 1). The median duration of IIAT was 13 days (IQR: 7-20 days).

Twenty patients were treated with TGC at $100 \mathrm{mg}$ every $12 \mathrm{~h}$ after a $200 \mathrm{mg}$ loading dose, and the other 11 patients were treated with TGC in an initial loading dose of $100 \mathrm{mg}$ followed by $50 \mathrm{mg}$ every $12 \mathrm{~h}$. Except for one patient who was treated with TGC monotherapy, the other 30 patients received combination therapy with cefoperazone-sulbactam 


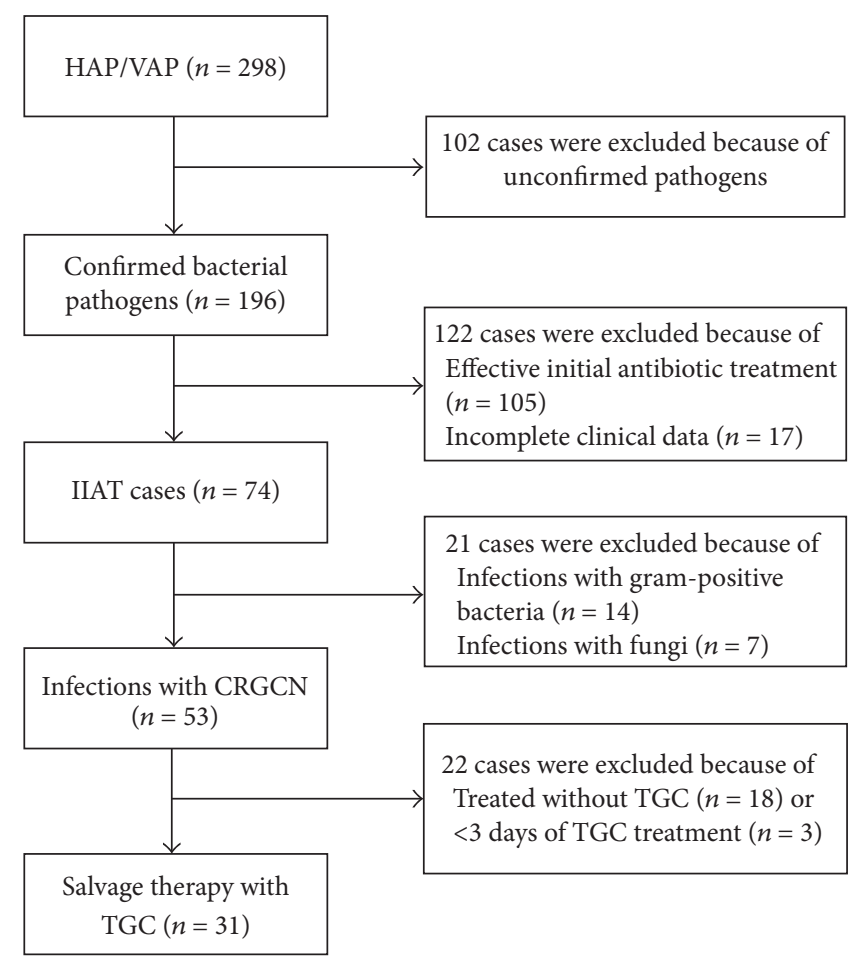

FIGURE 1: Study analysis populations. HAP: hospital-acquired pneumonia, VAP: ventilator-associated pneumonia, IIAT: inappropriate initial empiric antibiotic treatment, CRGNB: carbapenem-resistant Gram-negative bacteria, and TGC: tigecycline.

TABLE 2: Rates of resistance (\%) of the isolates to antimicrobial agents.

\begin{tabular}{|c|c|c|c|c|c|c|c|c|}
\hline \multirow[t]{2}{*}{ Antimicrobial } & \multicolumn{2}{|c|}{$\begin{array}{c}\text { Acinetobacter baumannii } \\
(n=21)\end{array}$} & \multicolumn{2}{|c|}{$\begin{array}{c}\text { Klebsiella pneumoniae } \\
(n=5)\end{array}$} & \multicolumn{2}{|c|}{$\begin{array}{l}\text { Escherichia coli } \\
\qquad(n=3)\end{array}$} & \multicolumn{2}{|c|}{$\begin{array}{l}\text { Stenotrophomonas maltophilia } \\
\qquad(n=2)\end{array}$} \\
\hline & $I^{*}$ & $R^{*}$ & $I$ & $R$ & $I$ & $R$ & $I$ & $R$ \\
\hline Ampicillin & 0 & 100 & & & & 100 & & \\
\hline Piperacillin-tazobactam & 9.5 & 76.2 & 0 & 80.0 & 33.3 & 66.7 & 0 & 100 \\
\hline Ceftriaxone & 0 & 100 & 0 & 100 & 0 & 100 & & \\
\hline Ceftazidime & 4.8 & 81.0 & 20.0 & 80.0 & 0 & 100 & 0 & 100 \\
\hline Cefepime & 19.0 & 76.2 & 20 & 60.0 & 33.3 & 66.7 & & \\
\hline Cefoperazone-Sulbactam & 14.3 & 476.2 & 0 & 100 & 33.3 & 66.7 & 0 & 100 \\
\hline Aztreonam & 28.6 & 71.4 & 0 & 80.0 & 0 & 66.7 & 0 & 100 \\
\hline Imipenem & 23.8 & 66.7 & 25.0 & 75.0 & 0 & 100 & 0 & 100 \\
\hline Meropenem & 28.6 & 61.9 & 0 & 100 & 33.3 & 66.7 & 0 & 100 \\
\hline Levofloxacin & 33.3 & 57.1 & 20 & 60.0 & 0 & 100 & & \\
\hline Minocycline & 28.6 & 52.4 & 20 & 60 & 0 & 66.7 & & \\
\hline Gentamycin & 14.3 & 47.6 & 0 & 50 & 0 & 100 & 50 & 50 \\
\hline SMZ-TMP & 0 & 47.6 & 0 & 80 & & & 0 & 50 \\
\hline TGC $^{*}$ & 4.8 & 0 & 0 & 0 & 0 & 0 & 0 & 0 \\
\hline
\end{tabular}

*TGC: tigecycline, $I$ : intermediate, and $R$ : resistance.

$(n=18,58.1 \%)$, piperacillin-tazobactam $(n=10,32.3 \%)$, or carbapenem $(n=3,9.7 \%)$. The mean duration of TGC therapy was $14.3 \pm 2.8$ days.

3.3. Clinical and Microbiological Outcomes. The clinical success rate of TGC therapy was $48.4 \%(n=15)$. TCG therapy failed in $16(51.6 \%)$ patients: ten patients died during TGC therapy, and six had to be switched to other antibiotics (piperacillin-tazobactam with amikacin and fosfomycin in four patients and meropenem with amikacin and fosfomycin in two patients) because their infection progressed after they had been treated with TGC for 5-11 days. The overall ICU 
mortality rate was $45.2 \%(n=14)$. One patient died because of a complicated pulmonary embolism, and the remaining thirteen died because of deteriorated pneumonia.

Microbiological eradication was achieved in 19 patients (61.3\%). Persistent positive culture results were found in the other 12 patients (38.7\%) at the end of TGC treatment (Table 1).

APACHE II scores were lower (15.0 versus 19.0, $P=0.04)$, the duration of IIAT was shorter ( 7 days versus 16 days, $P=$ 0.02 ), and the rate of microbiological eradication was higher (13 cases versus $6, P=0.009$ ) in successfully treated patients than in unsuccessfully treated patient. No differences were observed in demographic features, comorbidities, SOFA scores, antibiotics used prior to TGC, or the duration of TGC therapy (Table 1).

The clinical success rate was higher $[65.0 \%(13 / 20)$ versus $18.2 \%(2 / 11), P=0.023]$ and the ICU mortality rate was lower $[30.0 \%(6 / 20)$ versus $72.7 \%(8 / 11), P=0.031]$ in the patients who received a high-dose TGC therapy $(100 \mathrm{mg}$ every $12 \mathrm{~h}$ after a $200 \mathrm{mg}$ loading dose) than in patients who received a standard-dose TGC therapy (50 mg every $12 \mathrm{~h}$ after a $100 \mathrm{mg}$ loading dose). No significant difference was observed in the microbiological eradication rate between the two different doses of TGC therapy $[45.0 \%$ (9/20) versus $27.3 \%$ (3/11), $P=$ 0.452].

\section{Discussion}

In this study, we found that the clinical cure rate for patients treated with TGC regimens was $48.4 \%$ in a cohort of critically ill patients with nosocomial pneumonia who had received IIAT as a result of CRGNBs. The rate of microbiological eradication was $61.3 \%$ and the overall ICU mortality rate was $45.2 \%$. APACHE II scores were higher and IIAT durations were longer in unsuccessfully treated patients than in successfully treated patients. The high-dose TGC therapy $(100 \mathrm{mg}$ every $12 \mathrm{~h}$ after a $200 \mathrm{mg}$ loading dose) was superior to the standard-dose TGC therapy (50 mg every $12 \mathrm{~h}$ after a $100 \mathrm{mg}$ loading dose).

IIAT has been shown to significantly increase the likelihood of morbidity and mortality in patients with nosocomial pneumonia, and antibiotic resistance is the main cause of IIAT $[14,19,20]$. In Asia, the major isolates obtained from cases with HAP and VAP are nonfermenters (e.g., Acinetobacter and Pseudomonas aeruginosa) and Enterobacteriaceae [19, 20]. The rate of imipenem resistance in Acinetobacter is 67.3\% in Asian countries, while the rates for MDR and extensive drug resistance are $82 \%$ and $51.1 \%$, respectively. The MDR rate for Klebsiella pneumoniae is $44.7 \%$ [20]. Consistent with these data, in our study, $67.7 \%$ of the carbapenem-resistant isolates were A. baumannii, and the others were K. pneumoniae and E. coli.

Our study is novel because it was focused on patients with HAP/VAP who were treated with a TGC regimen as a salvage therapy subsequent to the failure of a previously selected antibiotic because of carbapenem-resistant pathogens. The off-label use of TGC has become widespread because of the scarcity of approved effective alternative antibiotics for MDR infections, such as nosocomial pneumonia, in addition to bloodstream and urinary tract infections [21]. In a few retrospective studies, TGC has demonstrated good efficacy against HAP and VAP caused by CRGNB. These studies have shown that TGC was effective in most patients (69.7-90\%) with VAP caused by carbapenem-resistant $A$. baumannii when used alone or in combination with other antimicrobials [12, 13, 22]. For E. coli and Klebsiella spp., which exhibit decreased susceptibility to carbapenems, the in vitro rates of susceptibility to TGC are $100 \%$ and $94.8 \%$, respectively. Nearly $70 \%$ of patients who are treated with TGC achieve resolution when an infection was caused by a carbapenemresistant or MDR Enterobacteriaceae [2].

A recent multicenter clinical study of VAP patients reported a significantly lower cure rate for a TGC regimen than was observed for an imipenem regimen [23]. However, only approximately $30 \%$ of the patients experienced a prior antibiotic failure in this previous study, and this rate is significantly different from the rate observed in our cohort. A meta-analysis including 13 randomized clinical trials demonstrated that TGC was associated with an increase in mortality and an increase in the noncure rate. These effects were independent of the infection type, trial design, and study size [7]. However, TGC has been prescribed in life-threatening infections for which there were few or no alternative agents [9-11, 15, 24]. A high-dose TGC regimen has been shown to result in better outcomes in patients with severe infections caused by MDR bacteria [15]. Our data also indicate that a high-dose TGC therapy is superior to a standard-dose TGC therapy. Pharmacokinetic-pharmacodynamic properties and patient-specific factors appear to offer one explanation for the observed differences in outcomes in TGC-treated patients with HAP. An inferior pharmacokinetic-pharmacodynamic index was observed in patients with poor clinical and microbiological responses [25]. Lower albumin concentrations, VAP status, worsening infections, or complications with baseline bacteremia have been associated with poor clinical outcomes $[25,26]$. Additionally, in our cohort, higher APACHE II scores and a longer duration of IIAT were observed in unsuccessfully treated patients.

Our study has several limitations. First, we performed a retrospective analysis that included a small number of patients. Second, in almost all of the patients, TGC was used in combination with other antibiotics. The small number of cases included in this study did not allow the use of a regression model that would separate the effect of TGC alone from the combined effects of the other antibiotics or the effects of the clinical characteristics, such as the APACHE II score. Finally, we did not monitor plasma or tissue concentrations of TGC. However, to the best of our knowledge, this is the first study to focus on TGC as a salvage regimen for HAP caused by CRGNB.

\section{Conclusions}

TGC, especially a high-dose regimen, might be a justifiable option in critically ill patients with nosocomial pneumonia caused by CRGNB who received IIAT for whom options are limited. Multicenter, prospective, randomized controlled clinical trials and pharmacokinetic research are required to 
confirm the role of TGC as an effective salvage treatment in critically ill patients with serious CRGNB infections.

\section{Competing Interests}

The authors declare that they have no competing interests.

\section{Authors' Contributions}

Xiaomai $\mathrm{Wu}$ drafted the protocol, performed the literature search, collected data, analyzed results, and drafted the manuscript. Yefei Zhu was involved in the conception, design, and coordination of the study. Liuyang Gong and Jian Lin were involved in data collection and the analyses of the results. Qiuying Chen and Dongqing Lv were involved in the conception and design of the study. Jiaxi Feng was involved in data collection and analysis. All authors read and approved the final manuscript.

\section{Acknowledgments}

This work was supported by the Science and Technology Foundation of Enze Medicine Center (no. 14EZD12).

\section{References}

[1] M. W. Olson, A. Ruzin, E. Feyfant, T. S. Rush III, J. O'Connell, and P. A. Bradford, "Functional, biophysical, and structural bases for antibacterial activity of tigecycline," Antimicrobial Agents and Chemotherapy, vol. 50, no. 6, pp. 2156-2166, 2006.

[2] T. Kelesidis, D. E. Karageorgopoulos, I. Kelesidis, and M. E. Falagas, "Tigecycline for the treatment of multidrug-resistant Enterobacteriaceae: a systematic review of the evidence from microbiological and clinical studies," Journal of Antimicrobial Chemotherapy, vol. 62, no. 5, pp. 895-904, 2008.

[3] B. Özbek and G. Ötük, "In vitro activities of tigecycline alone and in combination with colistin sulfate or sulbactam against carbapenem-susceptible and -resistant Acinetobacter baumannii strains isolated from Intensive Care Units," International Journal of Antimicrobial Agents, vol. 36, no. 2, pp. 191-192, 2010.

[4] H. S. Sader, D. J. Farrell, and R. N. Jones, "Tigecycline activity tested against multidrug-resistant Enterobacteriaceae and Acinetobacter spp. isolated in US medical centers (2005-2009)," Diagnostic Microbiology and Infectious Disease, vol. 69, no. 2, pp. 223-227, 2011.

[5] K. B. Waites, L. B. Duffy, and M. J. Dowzicky, "Antimicrobial susceptibility among pathogens collected from hospitalized patients in the United States and in vitro activity of tigecycline, a new glycylcycline antimicrobial," Antimicrobial Agents and Chemotherapy, vol. 50, no. 10, pp. 3479-3484, 2006.

[6] E. Tasina, A.-B. Haidich, S. Kokkali, and M. Arvanitidou, "Efficacy and safety of tigecycline for the treatment of infectious diseases: a meta-analysis," The Lancet Infectious Diseases, vol. 11, no. 11, pp. 834-844, 2011.

[7] P. Prasad, J. Sun, R. L. Danner, and C. Natanson, "Excess deaths associated with tigecycline after approval based on noninferiority trials," Clinical Infectious Diseases, vol. 54, no. 12, pp. 16991709, 2012.

[8] P. Montravers, H. Dupont, J.-P. Bedos, and P. Bret, "Tigecycline use in critically ill patients: a multicentre prospective observational study in the intensive care setting," Intensive Care Medicine, vol. 40, no. 7, pp. 988-997, 2014.

[9] B. Balandin Moreno, I. Fernández Simón, V. Pintado García et al., "Tigecycline therapy for infections due to carbapenemaseproducing Klebsiella pneumoniae in critically ill patients," Scandinavian Journal of Infectious Diseases, vol. 46, no. 3, pp. 175180, 2014.

[10] K. Pontikis, I. Karaiskos, S. Bastani et al., "Outcomes of critically ill intensive care unit patients treated with fosfomycin for infections due to pandrug-resistant and extensively drugresistant carbapenemase-producing Gram-negative bacteria," International Journal of Antimicrobial Agents, vol. 43, no. 1, pp. 52-59, 2014.

[11] M. Bassetti, C. Eckmann, K. F. Bodmann et al., "Prescription behaviours for tigecycline in real-life clinical practice from five European observational studies," Journal of Antimicrobial Chemotherapy, vol. 68, supplement 2, pp. II5-II14, 2013.

[12] R. Guner, I. Hasanoglu, S. Keske, A. K. Kalem, and M. A. Tasyaran, "Outcomes in patients infected with carbapenem-resistant Acinetobacter baumannii and treated with tigecycline alone or in combination therapy," Infection, vol. 39, no. 6, pp. 515-518, 2011.

[13] J. D. Chan, J. A. Graves, and T. H. Dellit, "Antimicrobial treatment and clinical outcomes of carbapenem-resistant Acinetobacter baumannii ventilator-associated pneumonia," Journal of Intensive Care Medicine, vol. 25, no. 6, pp. 343-348, 2010.

[14] M. K. Joung, K. T. Kwon, C.-I. Kang et al., "Impact of inappropriate antimicrobial therapy on outcome in patients with hospital-acquired pneumonia caused by Acinetobacter baumannii," Journal of Infection, vol. 61, no. 3, pp. 212-218, 2010.

[15] G. De Pascale, L. Montini, M. A. Pennisi et al., "High dose tigecycline in critically ill patients with severe infections due to multidrug-resistant bacteria," Critical Care, vol. 18, no. 3, article R90, 2014.

[16] N. Gupta, B. M. Limbago, J. B. Patel, and A. J. Kallen, "Carbapenem-resistant enterobacteriaceae: epidemiology and prevention," Clinical Infectious Diseases, vol. 53, no. 1, pp. 60-67, 2011.

[17] R. K. Shields, C. J. Clancy, L. M. Gillis et al., "Epidemiology, clinical characteristics and outcomes of extensively drug-resistant acinetobacter baumannii infections among solid organ transplant recipients," PLoS ONE, vol. 7, no. 12, article e52349, 2012.

[18] American Thoracic Society, "Guidelines for the management of adults with hospital-acquired, ventilator-associated, and healthcare-associated pneumonia," American Journal of Respiratory and Critical Care Medicine, vol. 171, no. 4, pp. 388-416, 2005.

[19] T. Kim, Y. P. Chong, S. Y. Park et al., "Risk factors for hospitalacquired pneumonia caused by carbapenem-resistant Gramnegative bacteria in critically ill patients: a multicenter study in Korea," Diagnostic Microbiology and Infectious Disease, vol. 78, no. 4, pp. 457-461, 2014.

[20] D. R. Chung, J.-H. Song, S. H. Kim et al., "High prevalence of multidrug-resistant nonfermenters in hospital-acquired pneumonia in Asia," American Journal of Respiratory and Critical Care Medicine, vol. 184, no. 12, pp. 1409-1417, 2011.

[21] M. E. Falagas, P. Lourida, P. Poulikakos, P. I. Rafailidis, and G. S. Tansarli, "Antibiotic treatment of infections due to carbapenemresistant enterobacteriaceae: systematic evaluation of the available evidence," Antimicrobial Agents and Chemotherapy, vol. 58, no. 2, pp. 654-663, 2014. 
[22] J. J. Schafer, D. A. Goff, K. B. Stevenson, and J. E. Mangino, "Early experience with tigecycline for ventilator-associated pneumonia and bacteremia caused by multidrug-resistant $\mathrm{Acl}$ netobacter baumannii," Pharmacotherapy, vol. 27, no. 7, pp. 980987, 2007.

[23] A. T. Freire, V. Melnyk, M. J. Kim et al., "Comparison of tigecycline with imipenem/cilastatin for the treatment of hospitalacquired pneumonia," Diagnostic Microbiology and Infectious Disease, vol. 68, no. 2, pp. 140-151, 2010.

[24] W. Kim, J. Moon, J. W. Huh et al., "Comparable efficacy of tigecycline versus colistin therapy for multidrug-resistant and extensively drug-resistant Acinetobacter baumannii pneumonia in critically Ill patients," PLoS ONE, vol. 11, no. 3, article e0150642, 2016.

[25] S. M. Bhavnani, C. M. Rubino, J. P. Hammel et al., "Pharmacological and patient-specific response determinants in patients with hospital-acquired pneumonia treated with tigecycline," Antimicrobial Agents and Chemotherapy, vol. 56, no. 2, pp. 10651072, 2012.

[26] P. C. McGovern, M. Wible, A. El-Tahtawy, P. Biswas, and R. D. Meyer, "All-cause mortality imbalance in the tigecycline phase 3 and 4 clinical trials," International Journal of Antimicrobial Agents, vol. 41, no. 5, pp. 463-467, 2013. 


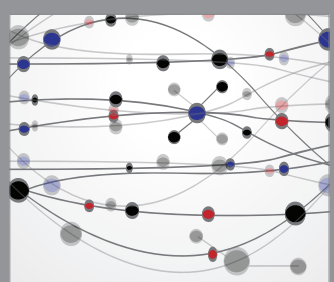

The Scientific World Journal
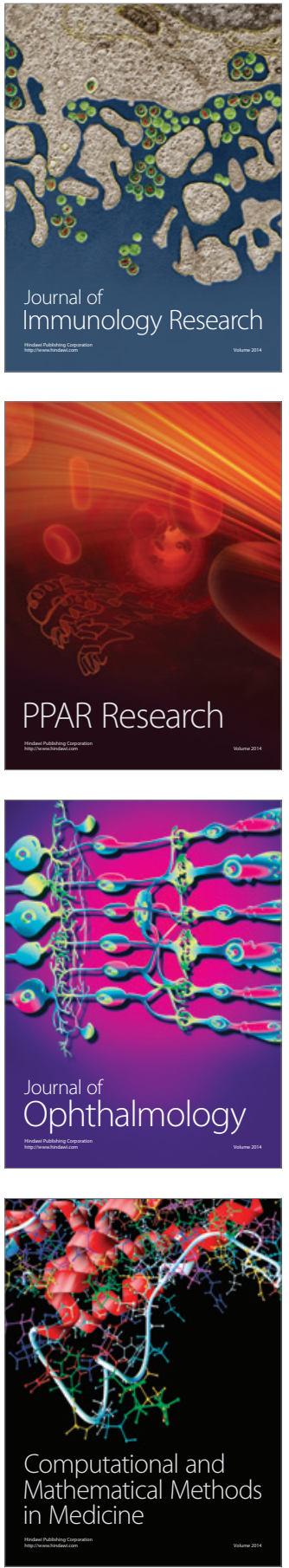

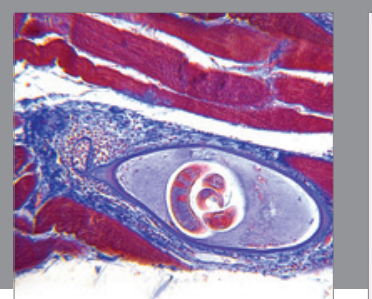

Gastroenterology Research and Practice

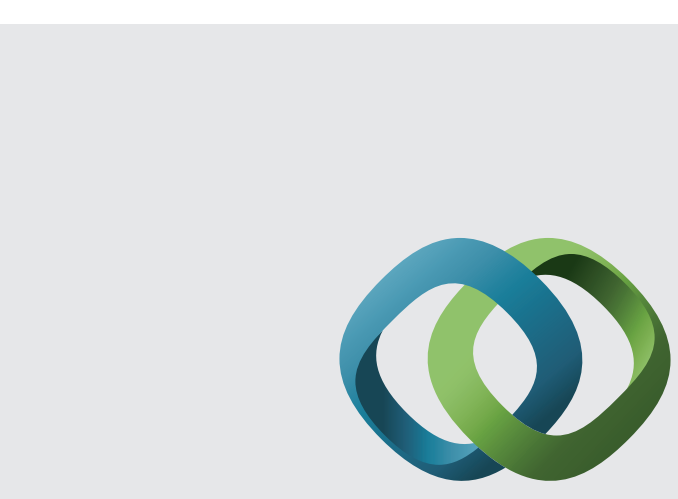

\section{Hindawi}

Submit your manuscripts at

http://www.hindawi.com
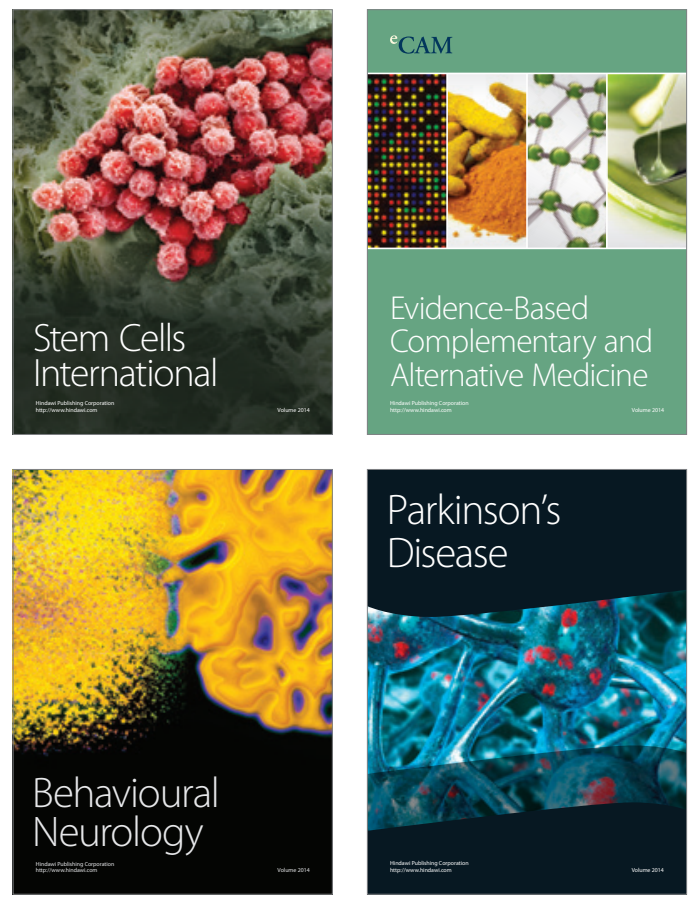
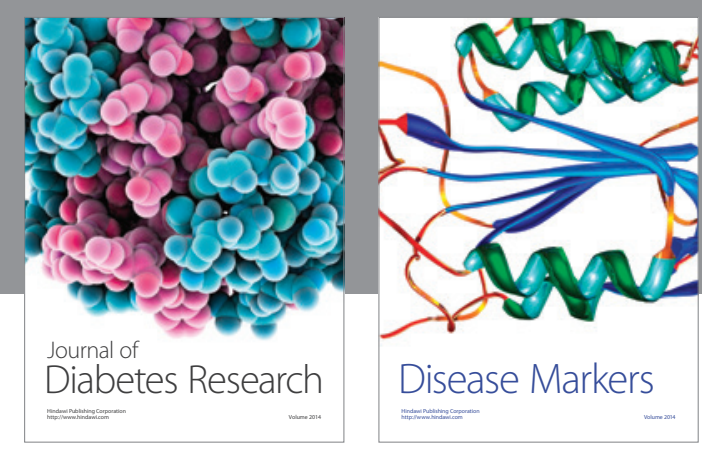

Disease Markers
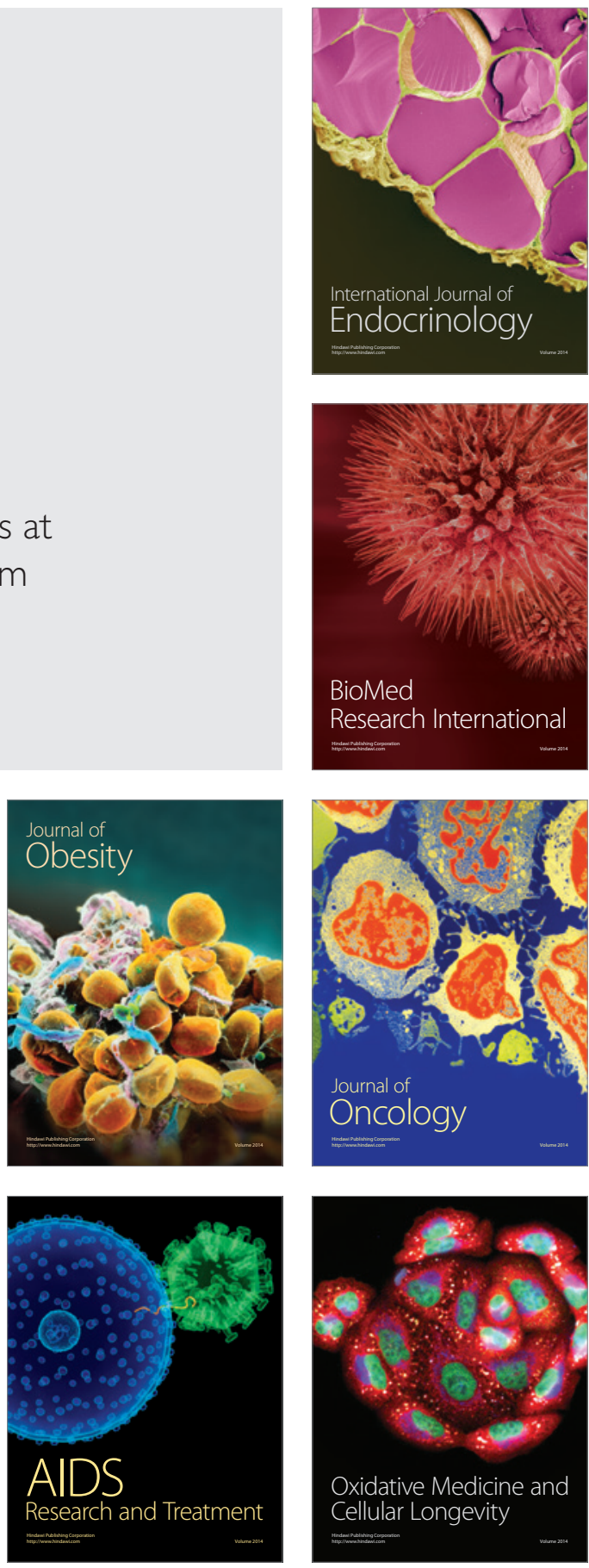